\title{
Modeling and Optimization of Carbon Steel AISI 1045 Surface Roughness in CNC Turning Based on Response Surface Methodology and Heuristic Optimization Algorithms
}

\author{
Vijay Nagandran ${ }^{1, ~ *, ~ T i a g r a j a h ~ V . ~ J a n a h i r a m a n ², ~ N o o r a z i a h ~ A h m a d ~}{ }^{3}$ \\ ${ }^{1}$ Department of Mechanical Engineering, Universiti Tenaga Nasional, Selangor, Malaysia \\ ${ }^{2}$ Department of Electronics and Communication Engineering, Universiti Tenaga Nasional, Selangor, Malaysia \\ ${ }^{3}$ Faculty of Creative Technology and Heritage, Universiti Malaysia Kelantan, Kelantan, Malaysia
}

Email address:

vijay_v24@yahoo.com (V. Nagandran)

${ }^{*}$ Corresponding author

\section{To cite this article:}

Vijay Nagandran, Tiagrajah V. Janahiraman, Nooraziah Ahmad. Modeling and Optimization of Carbon Steel AISI 1045 Surface Roughness in CNC Turning Based on Response Surface Methodology and Heuristic Optimization Algorithms. American Journal of Neural Networks and Applications. Vol. 3, No. 6, 2017, pp. 56-62. doi: 10.11648/j.ajnna.20170306.11

Received: October 30, 2017; Accepted: November 22, 2017; Published: January 11, 2018

\begin{abstract}
Surface roughness or surface quality is considered to be one of the most crucial requirement of a machined part since it directly influences the mechanical properties of the part. However, the traditional method of choosing cutting parameters' values to obtain a good surface finish has its own disadvantages. Therefore, an experimental study has been conducted to develop a suitable mathematical model and pair it with an optimization technique that able to produce low surface roughness of carbon steel AISI 1045. Response surface methodology (RSM) is used to develop the mathematical model whereas three types of heuristic optimization methods namely Genetics Algorithm (GA), Particle Swarm Optimization (PSO) and Simulated Annealing (SA) employed to optimize the model and find the optimal cutting parameters' values. A brief comparison of the three optimization methods has been made to study their performance to the developed model. Experimental results indicate that the proposed modeling technique and PSO are quite efficient in determining optimal cutting parameters for CNC turning of carbon steel AISI 1045.
\end{abstract}

Keywords: Carbon Steel AISI 1045, Genetic Algorithm, Particle Swarm Optimization, Response Surface Methodology, Simulated Annealing, Surface Roughness

\section{Introduction}

Surface finish is one of the most important technical requirements for a product in machining process. It has been characterized as the quality index of a product since it influences the mechanical performances such as frictional resistance and fatigue strength of the product. Besides, a product with good surface finish or low surface roughness eliminates the need for further machining. There are many factors such as cutting conditions, tool variables and workpiece variables that affect surface roughness. However, all these factors cannot be controlled to produce a product with good surface finish. A number of researchers have focussed on optimising cutting parameters mainly cutting speed, feed rate and depth of cut as these three input parameters are more controllable and have direct influence on the surface roughness of a machined product [1].

Current industry practise of choosing the desired cutting parameters is based on experience of a human process planner or from referring to the data in handbooks. The suggested data in handbooks are based on isolated laboratory tests using standard specimens. Many restrictive factors are carefully controlled here. Therefore, output from both human process planner and handbook data may not guarantee a desired surface roughness or produce some errors when machining in different environment conditions. This problem 
can be solved by finding the relationship between performance of the process (surface roughness) and its controllable input parameters (cutting parameters) by modeling the process through suitable mathematical techniques and optimizing using suitable optimization methods [2]. Moreover, by choosing and optimizing right technological parameter, machining time can be reduced and more number of items can be machined in a period [3].

Both traditional and non traditional optimization methods are employed in this field of study. However, a higher convergence speed to the global optimal solution and the ability to deal with non-linear complex machining problems had led heuristic optimization algorithms to be preferred over traditional optimization methods [4]. Examples of some heuristic algorithms are genetic algorithm, particle swarm optimization, simulated annealing and ant colony optimization $[5,6]$.

Chandrasekan et.al [7] in his study has stated that optimization techniques are necessary to obtain good surface roughness and dimensional properties. Following the statement he had developed a model to predict and analyze CNC turning of AISI 316 by employing RSM. His study revealed that the prediction values by RSM are fairly close with observed value. Apart from that, another study conducted by Azadi Moghaddam et. al [8] to study effect of input EDM parameters on surface quality of 2312 hot worked steel showed that employed GA considerably improved surface roughness. ANOVA was applied in this study and it proved that pulse on time and peak current significantly influence surface quality.

Previous work by Alimirzaloo [9] and Kalidass [10] had showed that RSM is one of the effective modeling method where it able to predict process parameters' values well in respect with surface roughness. Experimental investigation conducted by Shivade et.al [11] provided with results where head feed rate, peripheral speed of the part and emery force are the significant parameters that determines good surface roughness in finishing process of chrome coated printing cylinders. The both studies used ANOVA to verify the model adequacy.

Besides the field of machining, modeling and optimization has been employed in various field such as automotive, energy, environment studies and etc., where an attempt is made to predict the responses of pore-water pressure to rainfall using support vector machine (SVM). Generally, SVM performs in par with artificial neural network (ANN) [12]. A study conducted by Shukurillo Usmonov [13] is to find optimal characteristics of start-up variable frequency drive (VFD) based on different type of engines without modifying current control system to minimize energy losses. Genetics algorithm has been used for this purpose.

In this study, an attempt has been made by employing response surface methodology as an approach to develop a model which is then integrated with three non-conventional optimization methods in order to obtain optimal surface roughness for AISI 1045 carbon steel. Later, the three optimization methods are compared with each other to determine the best method for this study. AISI 1045 steel is commonly used in manufacturing industry due to its good machinability, weldability and high strength.

This paper is divided into six parts where part 2 and 3 discusses the theory of the modeling and optimization methods employed. Part 4 explains the experimental details and method used for collecting data, followed by part 5 which briefly discusses about the obtained results and part 6 concludes the study.

\section{Response Surface Methodology}

RSM employs both mathematical and statistical techniques which are useful for the modeling and analysis of problems where it quantifies the relationship between the measured response and the input factors (independent variables). The present work shows that a non-linear relationship exist between the surface roughness and the input factors which are cutting speed, feed rate and depth of cut.

There are two types of polynomial models which are first and second order. The polynomial model is usually referred as a regression model. The first order and second order or quadratic model is expressed by Equation (1) and Equation (2) respectively.

$$
\begin{aligned}
& y=\beta_{o}+\sum_{i=1}^{k} \beta_{i} x_{i}+\varepsilon \\
& y=\beta_{o}+\sum_{i=1}^{k} \beta_{i} x_{i}+\sum_{i=1}^{k} \beta_{i i} x_{i}^{2}+\sum_{i} \sum_{j} \beta_{i j} x_{i} x_{j}+\varepsilon
\end{aligned}
$$

where $\mathrm{y}$ denotes the response, $\beta_{o}$ is the constant and $\beta_{i,} \beta_{i i}, \beta_{i j}$ are the coefficients of the linear, quadratic and interaction terms respectively while $\varepsilon$ is the error term. The second order model is more effective in predicting the performance of the given data than first order model. Therefore, second order model is used in this study to develop the relationship between the process and the response.

\section{Optimization Methods}

\subsection{Genetics Algorithm}

Genetics algorithm is a heuristic search technique which imitates the processes that exist in nature. The technique is inspired by evolutionary biology such as inheritance, mutation, selection and cross over [14]. During each iteration, genetic algorithm maintains a population of potential individuals. These individuals are evaluated by their 'fitness'. A new population is then formed in the next iteration by selecting more fit individuals. Some members of this new population undergo alterations by means of crossover and mutation to form more potential solutions. After some number of generations, the program converges and best individual represents a optimal solution. This study has implemented GA in MATLAB to optimize the objective function of surface roughness. 


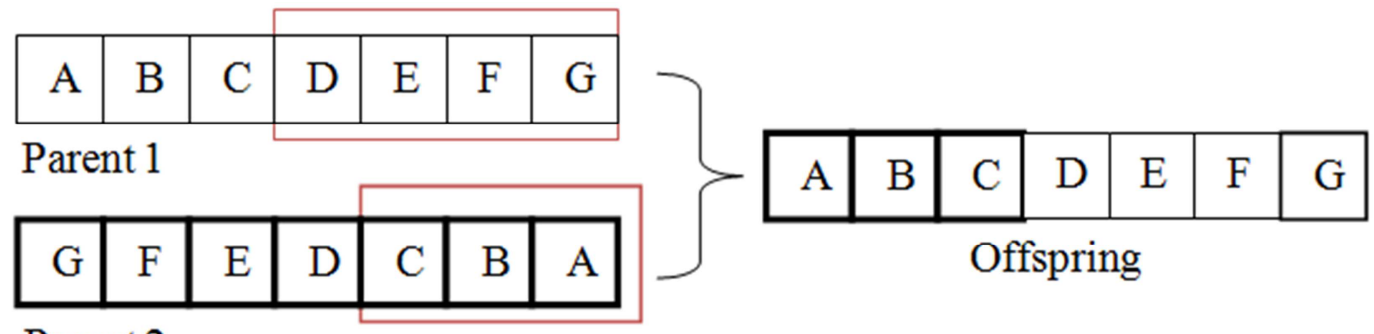

Parent 2

Figure 1. Crossover operation in GA [14].

Before mutation

After mutation
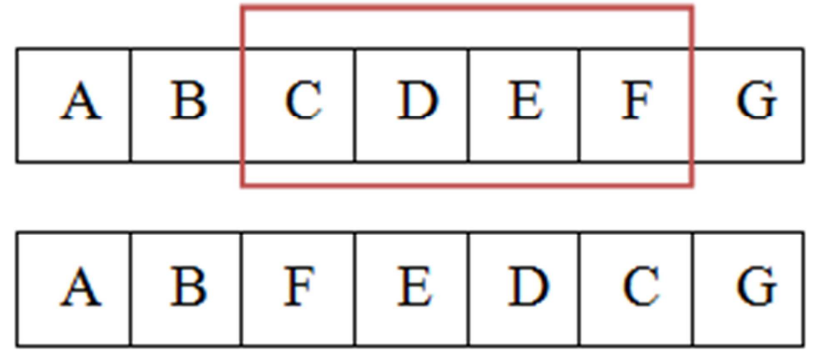

Figure 2. Mutation operation in GA [14].

\subsection{Particle Swarm Optimization}

Particle swarm optimization is a population based stochastic optimization technique developed by Eberhart and Kennedy [15, 16]. It is modeled on the social behaviours observed in animals such as birds, fish and certain species of which move in group [17]. PSO works by generating 'particles' randomly over the search space and at every iteration, each particle adjusts its velocity vector based on its momentum and the influence of its and neighbours best position. According to these factors, a new position is then computed. Optimal solution is obtained when the PSO converges after a certain number of generations.

\subsection{Simulated Annealing}

Simulated annealing is one of the earliest heuristic optimization method which is also nature inspired. The term annealing refers to the metallurgical process of heating a metal to a certain temperature followed by cooling the metal slowly at room temperature until the metal obtains a strong crystalline structure [18]. The SA algorithm works by initializing random points at first and a small random change is made to the current points by the perturbation mechanism. The objective function value of new solution is then calculated and compared with that of current solution. If the new state is better than the current state, the algorithm will accept it or else the acceptance probability of the new state will be smaller than 1 . This probability is affected by the parameters such as quality of movement and cooling schedule. Generally, the adopted cooling schedule influences the quality of solution found by the SA algorithm [19]. In this case, MATLAB's global optimization toolbox which contains SA solver was employed.

\section{Experimental Details}

The experiments were conducted on a $\mathrm{CNC}$ lathe machine with the tool holder MTJNR 2525M16 and a carbide insert cutting tool TNMG 160408-M3 TP2500. The workpiece used in this experiment is carbon steel AISI 1045 with diameter of $20 \mathrm{~mm}$ and length of $150 \mathrm{~mm}$. Each experiment was stopped after $100 \mathrm{~mm}$ of cutting length. The surface roughness of the workpiece was measured and recorded using SJ-301 surface roughness tester. The experimental data consist of 14 experiments which were used to develop models and train them while another different set of data which consist of 3 experiments were used for model validation.

Table 1. Chemical properties of carbon steel AISI 1045.

\begin{tabular}{ll}
\hline Element & Content (\%) \\
\hline Carbon, C & $0.43-0.50$ \\
Manganese, Mn & $0.60-0.90$ \\
Sulphur, S & $0.05(\max )$ \\
Phosphorus, P & $0.04(\max )$ \\
\hline
\end{tabular}

Table 2. Physical properties of carbon steel AISI 1045.

\begin{tabular}{ll}
\hline Properties & Metric \\
\hline Tensile Strength & $585 \mathrm{MPa}$ \\
Yield Strength & $450 \mathrm{MPa}$ \\
Modulus of Elasticity & $200 \mathrm{GPa}$ \\
Shear Modulus & $80 \mathrm{GPa}$ \\
Elongation at break & $12 \%$ \\
\hline
\end{tabular}

Box - Behnken design with quadratic model was used to plan the experiment in Minitab R17. BBD is the efficient response surface method which requires lowest number of experiments [20]. The design of experiment contains threelevel three input factors with full replication as shown in Table 3. 
Table 3. Machining parameters and their levels.

\begin{tabular}{llll}
\hline \multirow{2}{*}{ Parameters } & \multicolumn{2}{l}{ Level } & \\
\cline { 2 - 4 } & $\mathbf{- 1}$ & $\mathbf{0}$ & $\mathbf{1}$ \\
\hline Feed rate $(\mathrm{mm} / \mathrm{rev})$ & 0.1 & 0.2 & 0.3 \\
Cutting speed $(\mathrm{m} / \mathrm{min})$ & 100 & 200 & 300 \\
Depth of cut $(\mathrm{mm})$ & 0.1 & 0.8 & 1.5 \\
\hline
\end{tabular}

The experiment was conducted as per the design matrix and resulting data were collected. The following Table 4 shows the obtained experimental data.

Table 4. Experimental layout for the Box-Behnken Design.

\begin{tabular}{lllll}
\hline No. & Feed rate & Cutting Speed & Depth of Cut & SR \\
\hline 1 & 0.2 & 100 & 1.5 & 1.62 \\
2 & 0.3 & 200 & 0.1 & 3.54 \\
3 & 0.1 & 300 & 0.8 & 0.77 \\
4 & 0.1 & 200 & 1.5 & 0.76 \\
5 & 0.2 & 300 & 0.1 & 1.47 \\
6 & 0.2 & 200 & 0.8 & 1.27 \\
7 & 0.2 & 300 & 1.5 & 1.46 \\
8 & 0.1 & 100 & 0.8 & 0.59 \\
9 & 0.2 & 200 & 0.8 & 1.37 \\
10 & 0.3 & 100 & 0.8 & 3.42 \\
11 & 0.3 & 300 & 0.8 & 3.20 \\
12 & 0.2 & 200 & 0.8 & 1.58 \\
13 & 0.3 & 200 & 1.5 & 3.35 \\
14 & 0.2 & 100 & 0.1 & 1.82 \\
\hline
\end{tabular}

\section{Results and Discussion}

\subsection{Modeling}

The coefficients of the second order model as shown in Equation (2) were determined using the statistical software Minitab R17. The developed mathematical model for $R_{a}$ is given in Equation (3). Up to 5 decimal points were taken to ensure a better accuracy of the equation. where $A$ is feed rate, $B$ is cutting speed and $C$ is depth of cut. The RSM model shown in Equation (3) can be used to predict the surface roughness by introducing a new set of input parameters' values. The RSM model is then evaluated based on the difference between the actual surface roughness readings and the predicted value. The difference is presented in terms of mean squared error (MSE), root mean square error (RMSE) and mean absolute percentage error (MAPE). The calculations were performed using Equations (4), (5) and (6).

$$
\begin{gathered}
\text { MSE }=\frac{1}{\mathrm{n}} \sum_{1}^{\mathrm{i}}\left(\mathrm{T}_{\mathrm{i}}-\mathrm{y}_{\mathrm{i}}\right)^{2} \\
\text { RMSE }=\sqrt{\frac{1}{\mathrm{n}} \sum_{1}^{\mathrm{i}}\left(\mathrm{T}_{\mathrm{i}}-\mathrm{y}_{\mathrm{i}}\right)^{2}} \\
\text { MAPE }=\frac{1}{\mathrm{n}} \sum_{1}^{\mathrm{i}}\left[\frac{\mathrm{T}_{\mathrm{i}}-\mathrm{y}_{\mathrm{i}}}{\mathrm{T}_{\mathrm{i}}}\right] \times 100
\end{gathered}
$$

where $n$ is the number of samples, $T_{i}$ is the measured value and $y_{i}$ is the predicted output. The results are presented in the following Table 5.

Table 5. Model evaluation results.

\begin{tabular}{ll}
\hline Evaluation Method & Value \\
\hline MSE & 0.008026 \\
RMSE & 0.089589 \\
MAPE & $5.632116 \%$ \\
\hline
\end{tabular}

The low error output shows that the developed model has good performance and able to predict the surface roughness well. The obtained RSM model is then used as the objective function for optimization algorithms to find the optimal cutting parameters.

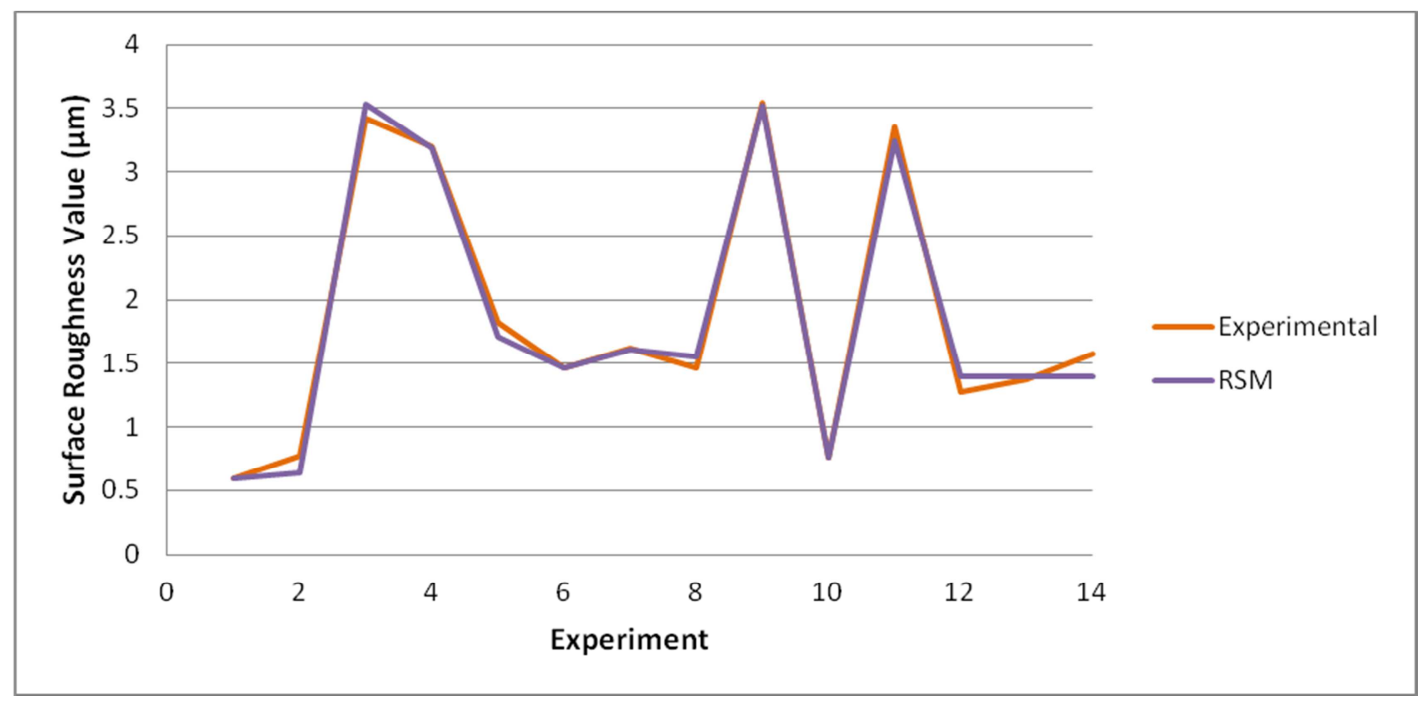

Figure 3. Comparison of predicted and experimental values. 


\subsection{ANOVA}

Analysis of variance (ANOVA) was conducted on the developed RSM model by Minitab to understand the influence of each parameter on the process and to verify the adequacy of the model. Table 6 shows us the ANOVA output for the developed RSM model.

Table 6. ANOVA output.

\begin{tabular}{lllll}
\hline Interaction & S.S & M.S & F-value & P-value \\
\hline $\begin{array}{l}\text { Model } \\
\text { Linear }\end{array}$ & 16.1692 & 1.79657 & 75.36 & 0.000 \\
A & & & & \\
B & 0.0108 & 0.01078 & 0.45 & 0.531 \\
$\mathrm{C}$ & 0.0160 & 0.01597 & 0.67 & 0.450 \\
Square & 0.0016 & 0.00161 & 0.07 & 0.805 \\
$\mathrm{~A} * \mathrm{~A}$ & & & & \\
$\mathrm{~B} B \mathrm{~B}$ & 0.0246 & 0.02463 & 1.03 & 0.356 \\
$\mathrm{C} * \mathrm{C}$ & 0.9473 & 0.94786 & 39.76 & 0.001 \\
2-Way & 0.0401 & 0.04006 & 1.68 & 0.251 \\
$\mathrm{~A} * \mathrm{~B}$ & & & & \\
$\mathrm{~A}{ }^{*} \mathrm{C}$ & 0.0400 & 0.04000 & 1.68 & 0.252 \\
$\mathrm{~B} \mathrm{C}$ & 0.0090 & 0.00903 & 0.38 & 0.565 \\
\hline
\end{tabular}

Since developed RSM model is full quadratic, it contains linear, square and two-way interaction in its equation. S.S, M.S and F-value are used to calculate the P-value for a term. The P-value is a probability that measures and determines whether the terms and model are statistically significant. The lower P-value than $\alpha$ denotes that the model is significant and provides stronger evidence against the null hypothesis. The value of $\alpha$ is 0.05 . The higher F-value and lower P-value for linear term A (feed rate) compared to B (cutting speed) and C (depth of cut) tells that feed rate has more significance and associated with the response (surface roughness).

Table 6 below shows the regression statistics. The $\mathrm{R}^{2}$ value of $99.27 \%$ shows that the model is good at fitting data. This means that data points fall closer to the fitted regression line. Besides, the model describes the response very well with a smaller standard deviation, $\mathrm{S}$ and has a good predictability with high $\mathrm{R}^{2}$ (pred).

Table 7. Regression Statistics.

\begin{tabular}{ll}
\hline Parameters & Values \\
\hline $\mathrm{S}$ & 0.154397 \\
$\mathrm{R}^{2}$ & $99.27 \%$ \\
$\mathrm{R}^{2}$ (adj) & $97.95 \%$ \\
$\mathrm{R}^{2}$ (pred) & $92.52 \%$ \\
\hline
\end{tabular}

\subsection{Optimization}

Three different heuristic optimization methods namely GA, PSO and SA were used to minimize the surface roughness and find the optimal values of cutting parameters. Following is the objective and constraint for the optimization algorithms.

Minimize $\mathrm{R}_{\mathrm{a}}$ with subjected to

$100 \mathrm{~mm} / \mathrm{rev} \leq \mathrm{A} \leq 300 \mathrm{~mm} / \mathrm{rev}$

$0.1 \mathrm{~m} / \mathrm{s} \leq \mathrm{B} \leq 0.3 \mathrm{~m} / \mathrm{s}$

\section{$0.1 \mathrm{~mm} \leq \mathrm{C} \leq 1.5 \mathrm{~mm}$}

All optimizations were conducted using MATLAB software and the main parameters of each optimization algorithm were fine tuned by performing a series of experimental trials to ensure optimal results are being produced. The parameters and their values are shown in Tables 8,9 and 10 .

Table 8. GA parameters and their values.

\begin{tabular}{ll}
\hline Parameters & Values \\
\hline Population Size & 50 \\
Scaling Function & Rank \\
Selection Function & Stochastic uniform \\
Reproduction Count & 2.5 \\
Crossover Function & Heuristic \\
Crossover Fraction & 0.8 \\
Mutation Function & Constraint dependent \\
Generations & 300 \\
\hline
\end{tabular}

Table 9. PSO parameters and their values.

\begin{tabular}{ll}
\hline Parameters & Values \\
\hline Population Size & 50 \\
Generations & 300 \\
Constraint Boundary & penalize \\
Social Attraction & 1.25 \\
Cognitive Attraction & 0.5 \\
\hline
\end{tabular}

Table 10. SA parameters and their values.

\begin{tabular}{ll}
\hline Parameters & Values \\
\hline Max function evaluations & 9000 \\
Stall iterations & 9000 \\
Temperature update function & Exponential temperature update \\
Annealing function & Fast annealing \\
\hline
\end{tabular}

The output of these three optimization methods were compared and analyzed to determine the best optimization method for the developed model. Since these three heuristic optimization algorithms employ random starting points, they produce different output for each run. Therefore, a total of 30 runs were generated and the average values are tabulated in the following Table 11.

Table 11. Comparison of output of three optimization methods.

\begin{tabular}{lllll}
\hline Algorithm & A & B & C & $\mathbf{R}_{\mathbf{a}}$ \\
\hline GA & 202.6230 & 0.1 & 0.38 & 0.497903 \\
PSO & 202.6226 & 0.1 & 0.38 & 0.497900 \\
SA & 203.3270 & 0.1 & 0.38 & 0.498066 \\
\hline
\end{tabular}

Based on the result, PSO and GA performed better than SA and produced almost the same output. Both PSO and GA performed $0.03 \%$ better than SA. In terms of time taken, PSO tends to converge faster than GA in order to get to the global solution. 


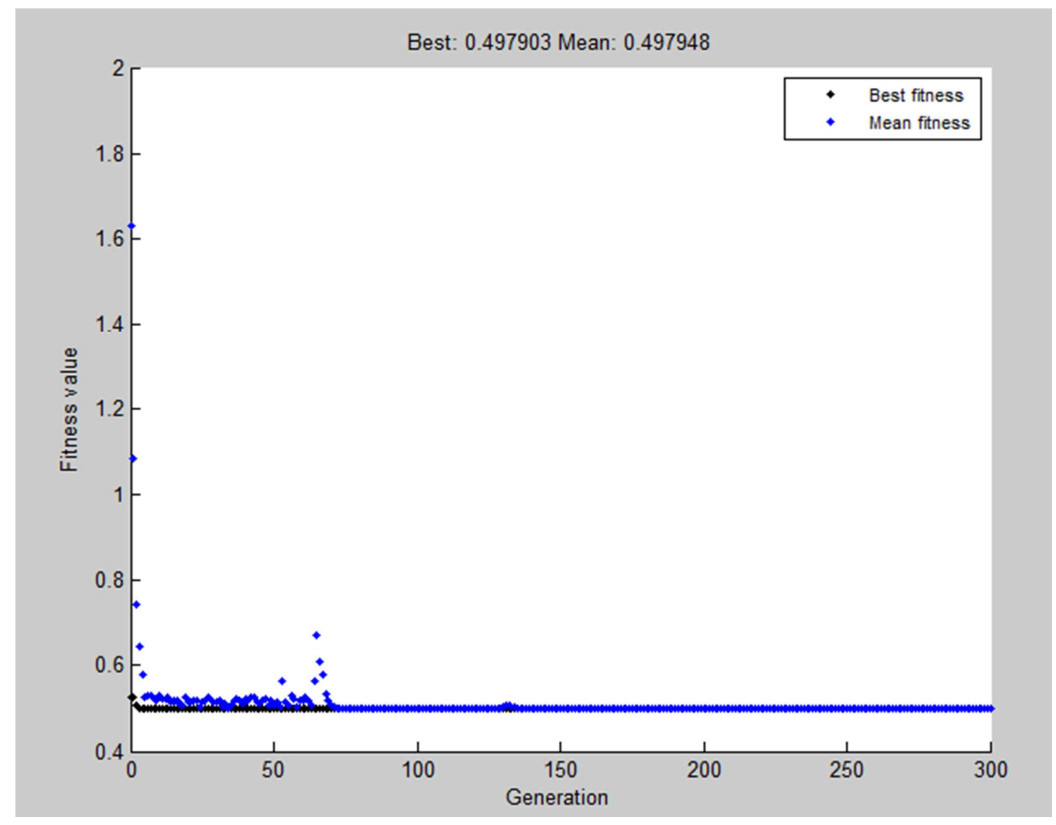

Figure 4. GA convergence plot.

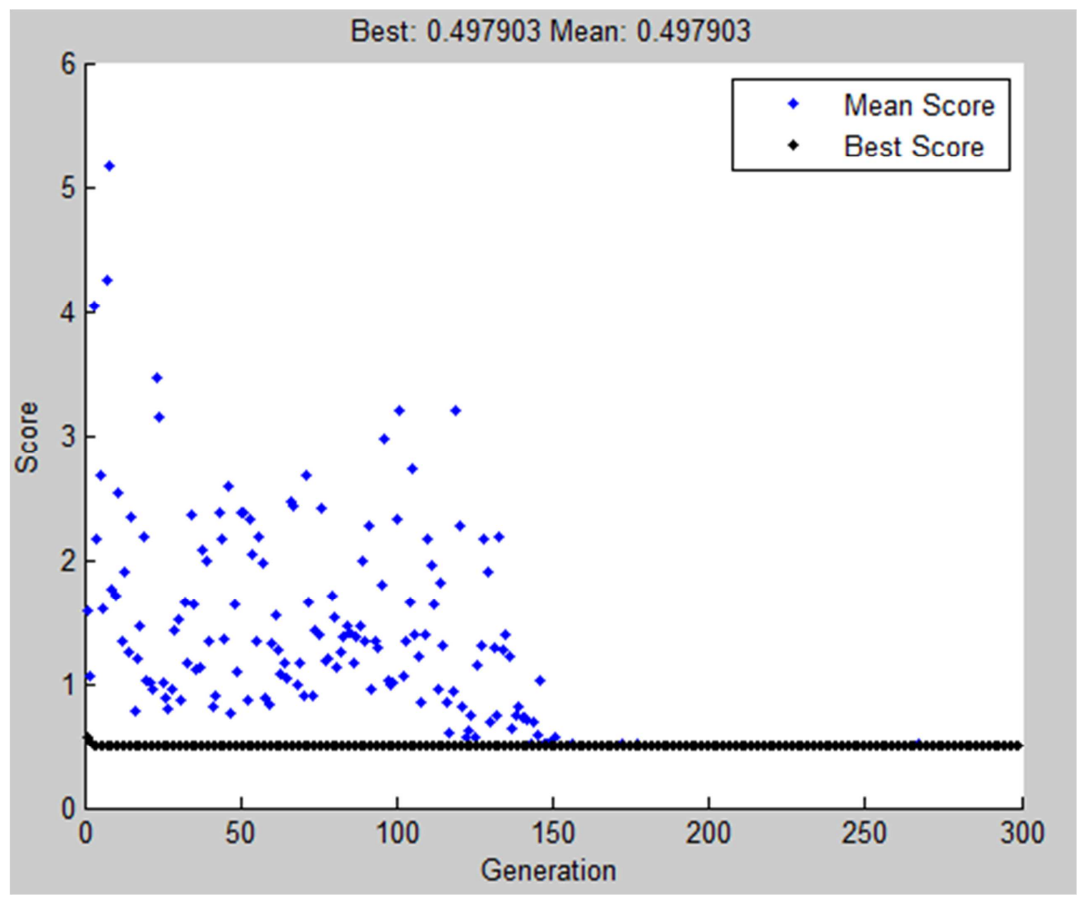

Figure 5. PSO convergence plot.

\section{Conclusion}

In this paper, experiment was conducted to develop a RSM model that able to predict surface roughness of carbon steel AISI 1045. From the model evaluation and ANOVA output, it was found out that the model able to predict surface roughness in terms of cutting parameters quite well. The model was then optimized with three types of heuristic optimization methods using MATLAB to compare the output and performance of them. After the optimization, it is concluded that:- (i) PSO and GA generally performed well and better than SA by producing lower surface roughness.

(ii) PSO converges and produces output at faster rate compared to GA \& SA.

Therefore, from this study, it is known that PSO is a better heuristic optimization method than GA \& SA. However, this study posses certain limitations where all three optimization methods employ random starting points and may produce different output each time and the obtained simulation results need to be verified by machining with the obtained values to determine the accuracy of the optimization output. 


\section{References}

[1] Makadia, A. J., and Nanavati, J. I., "Optimization of Machining Parameters for Turning Operations Based on Response Surface Methodology", Measurement, Vol. 46, No. 4, (2012), 1521-1529.

[2] Rao, V., "Advanced Modeling and Optimization of manufacturing processes", London, Springer, (2011).

[3] Sandor, B., and Bel., K., "Lean Production Planning for 5 Axes CNC Driven Milling Machine", Machine Learning Research, Vol.2, No.2, (2017), 66-72.

[4] Yang, X. S., "Engineering Optimization: An Introduction with Metaheuristic Applications", New York, John Wiley and Sons Inc, (2010).

[5] Gao, W. F., and Liu, S. Y., "A Modified Artificial Bee Colony", Computers \& Operations Research, Vol. 39, No. 2, (2012), 687-697.

[6] Haupt, R. L., and Koehler, A. B., "Practical Genetic Algorithms", New York, John Wiley and Sons Inc, (1998).

[7] Chandrasekaran, K., Marimuthu, P., and Raja, K., "Prediction Model for CNC Turning on AISI316 with Single and Multilayered Cutting Tool Using Box Behnken Design", International Journal of Engineering, Transactions A: Basics, Vol. 26, No. 4, (2013), 401-410.

[8] Azadi Moghaddam, M., and Kolahan, F., "Modeling and Optimization of Surface Roughness of AISI2312 Hot Worked Steel in EDM based on Mathematical Modeling and Genetic Algorithm", International Journal of Engineering, Transactions C: Aspects, Vol. 27, No. 3, (2014), 417-424.

[9] Alimirzaloo, V., Modanloo, V., and Babazadeh Asbagh, E., "Experimental Investigation of the Effect of Process Parameters on the Surface Roughness in Finishing Process of Chrome Coated Printing Cylinders", International Journal of Engineering, Transactions C: Aspects, Vol. 29, No. 12, (2016), 1775-1782.

[10] Kalidass, S., and Mathavaraj Ravikumar, T., "Cutting Force Prediction in End Milling Process of AISI 304 Steel Using Solid Carbide Tools", International Journal of Engineering, Transactions A: Basics, Vol. 28, No. 7, (2015), 1074-1081.

[11] Shivade, A. S., Bhagat, S., Jagdale, S., Nikam, A., and Londhe, P., "Optimization of Machining Parameters for Turning Using Taguchi Approach", International Journal of Recent Technology and Engineering, Vol. 3, No. 1, (2014), 145-149.

[12] Khamaruzaman, Y., Nuraddeen, B., Muhammad, M., and Mohamed, I., "Linear Kernel Support Vector Machines for Modeling Pore-Water Pressure Responses", Journal of Engineering Science and Technology, Vol.12, No.8, (2017), 2202-2212.

[13] Shukurillo, U., "Optimization of the Launching Process in the Electric Drive with the Help of Genetic Algorithm", Machine Learning Research, Vol.2, No.2, (2017), 61-65.

[14] Michalewicz, Z., "Genetics Algorithm + Data Structures = Evolution Programs ( $3^{\text {rd }}$ ed.)", New York, Springer, (1996).

[15] Eberhart, R. C., and Kennedy, J., "A New Optimizer Using Particles Swarm Theory" in $6^{\text {th }}$ International Symposium on Micro Machine and Human Science, Nagoya, Japan, 39-43. (1995).

[16] Eberhart, R. C., and Kennedy, J., "Particle swarm Optimization" in IEEE International Conference on Neural Network, Perth, Australia, 1942-1948, (1995).

[17] Bidya Prakash, M., and Shatendra, S., "A Review on Application of Bio-Geography Based Algorithm and Other Optimization Techniques, International Journal of Management, Information, Technology and Engineering, Vol. 3, No. 6, (2015), 19-28.

[18] Majhi, B. P., and Sahu, S., "A Review on Application of Biogeography Based Algorithm and Other Optimization Techniques", International Journal of Management, Information Technology and Engineering, Vol. 3, No. 6, (2015), 19-28.

[19] Balram, S., "Study of Simulated Annealing Based Algorithms for Multiobjective Optimization of a Constrained Problem", Computers and Chemical Engineering, Vol. 28, No. 10, (2004), 1849-1871.

[20] Nooraziah, A., and Tiagrajah, V. J., "A Study on Regression Model Using Response Surface Methodology", Applied Mechanics and Materials, Vol. 66, No. 6, (2014), 235-239. 